\title{
Abstract Interpretation
}

\section{CS252r Spring 2011}

Slides from

Principles of Program Analysis by Nielson, Nielson, and Hankin 


\section{Reference}

- Slides 2-16, 37-58 from Chap. 4 of Principles of Program Analysis by Nielson, Nielson, and Hankin http://www.imm.dtu.dk/ riis/PPA/slides4.pdf 